\title{
Learning by Living: Empathy Learned through an Extended Medical Education Immersion Project
}

\author{
Ianna Hondros-McCarthy, Philip Barber, Marilyn R. Gugliucci \\ University of New England College of Osteopathic Medicine, Biddeford, ME, USA \\ Email:mgugliucci@une.edu
}

Received 12 February 2016; accepted 25 March 2016; published 28 March 2016

Copyright (C) 2016 by authors and Scientific Research Publishing Inc.

This work is licensed under the Creative Commons Attribution International License (CC BY). http://creativecommons.org/licenses/by/4.0/

c) (i) Open Access

\begin{abstract}
Introduction: Medical student training and experiences in nursing homes are often viewed as negative; however, long-term care services represent a growing aspect of our medical system that receives little attention in medical education. The University of New England College of Osteopathic Medicine Learning by Living Nursing Home Immersion Project accelerates students learning about older adult care and the importance of empathy. Methods: Learning by Living applies qualitative ethnographic/autobiographic research methods to answer the question: "What is it like for me to live the life of an older adult nursing home resident?" Two first year medical students (female, 25 y/o \& male, $27 \mathrm{y} / 0$ ) were "admitted" into two different nursing homes for 11 days each. The students were wheelchair reliant from their assigned diagnoses of dominant-side paralysis secondary to stroke and aspiration pneumonia; requiring standard procedures of care experienced by older adults residents with similar conditions. Data were collected in the form of journal notes for pre-fieldwork, fieldwork, and post-fieldwork and included subjective and objective reporting of observations, experiences, and resident encounters. Analyses included journal review and thematic categorization and coding through content analysis. Results: Four themes of significance intersected for the two medical students: 1) control; 2) quality of life; 3) communication; and 4) isolation. Being immersed in nursing home life provided the students with firsthand experiences of loss and challenges faced by nursing home residents. This aided students' learning about empathy and communication as key components of providing health care. Conclusion: This experience imparted knowledge to the medical students' about aging, dignity, and the importance of making meaningful connections in order to thrive. These insights provided a gateway to establish a model of patient-centered care that is comprehensive and empathic for the older adult population.
\end{abstract}

\section{Keywords}

Empathy, Nursing Home Immersion, Experiential Medical Education, Translational Medical

How to cite this paper: Hondros-McCarthy, I., Barber, P. and Gugliucci, M.R. (2016) Learning by Living: Empathy Learned through an Extended Medical Education Immersion Project. International Journal of Clinical Medicine, 7, 236-244. 


\section{Practice}

\section{Introduction}

The purpose of the University of New England College of Osteopathic Medicine (UNECOM) Learning by Living Nursing Home Immersion Project (heretofore referred to as Learning by Living) is to provide medical students in training with first-hand experience of living the life of an elder to answer the question: "What is it like for me to live the life of an older adult nursing home resident?" Medical students state that this extended immersion learning opportunity is life altering for them and enhances their skills to become better physicians [1]. For this article, the student authors focused on their renewed learning about empathy and its role in patient care.

\subsection{Learning by Living Defined}

The Learning by Living project is based on qualitative research, whereby a "culture" is observed by the researcher (medical student) living within a unique environment (nursing home). Life experiences of the medical students while living in the environment as an older adult resident are reported. The Project was designed and implemented by Dr. Marilyn R. Gugliucci in 2005 and since this time, medical students have been "admitted" into Nursing Homes to Live the Life of an Elder Nursing Home Resident for 2 weeks-24/7-complete with a medical diagnosis and standard procedures of care [1]. Specifically, these students were assigned a diagnosis which made them each wheelchair reliant (stroke with dominant side weakness) and subject to eating a pureed food diet (aspiration pneumonia). Their standard procedures of care included being toileted, bathed, transferred, and administered medications (M\&Ms) on a regular schedule.

There are three stages of the Learning by Living ethnographic immersion: 1) pre-fieldwork-Getting ready for the experience; 2) fieldwork—Living in the environment, which includes a diagnosis, "medication" regimen, group and solitary meals, and engaging in relationship building; and 3) post-fieldwork-Reflection on what occurred during fieldwork [3] [4].

Traditional medical education methods may include short visits to the nursing home with quick exits. Medical student training and experiences in nursing homes are often viewed as negative, which mirrors the views expressed by the general public [2]. However, it is important to note that as stated by White (2008), "long-term care services represent a growing aspect of our medical system that receives little attention in medical education" [2]. The truth is, in the United States, nursing homes are often referred to as "institutions," which conjures up a depressing image and receives negative attention. Learning by Living has been designed with these aforementioned views in mind and is based on three significant premises to augment medical education within nursing homes: 1) older adults residing in nursing homes are human beings deserving of good care, respect, and "community" connection; 2) medical students can attain medical care skills including empathy and advanced relationship building skills with older adults from living the life of an older adult nursing home resident for an extended period of time; and 3) older adults and their care partners have perceptions and considerations that are relevant to the health care process. Additionally, living in a nursing home enables medical students to build knowledge, skills, and attitudes about patient care by constructively adapting to this environment through experiences and reflection [1]. In doing so, students become well equipped to form patient-centered care models based on the care they received and preferred. Each stage of this immersion research contributed to the medical students' learning about empathy; an often lost art in medical education.

\subsection{Empathy in Medical Education}

Research during the past 10 years has revealed that empathy in osteopathic medical students and residents tends to erode during medical school and residency training [5]. The somewhat reductive process of learning the body's vital systems can distract students from a more holistic approach to their patients. Yet research findings in health the health professions training indicates that empathy and the ability to help other people are directly correlated, and empathy can lead to more positive clinical outcomes [6]. Treating the constellation of symptoms that patients, especially older patients, present with can be to the detriment to their healing process. Feedback from patients has also indicated that they have a more positive treatment experience when they feel their physi- 
cian's care is rooted in empathy [6].

Learning by Living has a variety of medical education pearls; however the medical students determined that this extended immersion learning project advanced their understanding of empathy. For this article, empathy is defined as an attribute of a successful physician consisting of: a) understanding a patient's perspective with the intention to help them; and b) the ability to communicate that understanding to the patient in their clinical interactions [1]. Living as a nursing home resident raised mindfulness about empathy and its significance as a physician skill. Regardless of fields of medical practice these students select, empathy is one attribute they want to utilize when working with their future patients.

\section{Methodology}

\subsection{Research Design}

Learning by Living applies qualitative ethnographic/autobiographic research methods based in phenomenology [4] [7]-[10]. This methodological approach, through immersion into a culture (ethnography), creates structures of experience and raises consciousness. In essence, these students' became aware of their perspectives and experiences about life lived as an older adult nursing home resident and wrote about these (auto-biographic) so that interpretations were related to "self" rather than externally focused on the nursing home residents, staff, or nursing home practices [1]. This project was submitted for IRB approval and was deemed exempt as the researchers are adult medical students who volunteer to be "admitted" into a nursing home. No ethical issues have arisen from conducting this research. These medical students have current training in HIPAA and background checks were completed prior to beginning their medical education.

\subsection{Participants and Research Phases}

Two first year University of New England College of Osteopathic Medicine students (25 y/o female and 27 y/o male) volunteered to conduct this non-credit Learning by Living immersion research during the summer of 2015 (since its inception Learning by Living has been a volunteer project for students and nursing homes). They were selected from a group of first year medical students who applied to conduct this research. Selection was made by the project principal investigator based on an application whereby each student wrote an essay on why s/he wanted to conduct this research and what made the students most suitable for living in a nursing home. The two students selected exemplified a drive to expand their understanding of older adult patient care and empathy, both expressed strong work ethics, and both students had a passion for self-improvement.

There are approximately twenty nursing homes in four states who have participated in this project. The principal investigator developed a rotating system for asking nursing homes to participate so as to not over use any nursing home. The two nursing homes that were asked agreed to participate, one in New Hampshire and one in Maine; both were non-profit duel eligible homes with approximately 100 residents each. The nursing homes work with the Learning by Living project principal investigator to ensure there was a bed available and the staffs were willing to provide standard procedures of care to the student. Admission and discharge dates were negotiated with the nursing home administrator and each student. Students had significant responsibilities within each of the aforementioned phases of the Learning by Living project: 1) pre-field work; 2) field work; and 3) post-field work.

\subsubsection{Pre-Fieldwork}

(Prior to nursing home admission): Students completed a registration form that included demographic data, an essay on their interest in living in a nursing home, and a list of assumptions each student believed to be true about older adult nursing home residents and the nursing homes environment. They were then oriented to the project that included details on the Learning by Living project, information about the research design and data collection methods, medical diagnoses, and the support systems for the student (the Project PI was considered to be the "adult daughter"). A preparation document provided information on what clothes to pack, items to bring that would aid in creating a feeling of home, and information about data collection. Additionally, each student wrote their 85 year-old persona and life review that included such details as marriage, children, profession, and a back story for their diagnoses. This guided social/medical history aided the students in answering questions during admission into the nursing home. Students began journaling (pre-field notes) at the close of this meeting, reflecting on friends and family reactions to their being admitted into a nursing home, thoughts/feelings about 
their own admission into a home, deciding what to do with pets and/or possessions, and thoughts on leaving their own homes [1].

\subsubsection{Fieldwork}

(Living in the nursing home): The Project PI drove the students to their respective nursing homes on the day of admission. Students were admitted as if they had been released from the hospital emergency room; no pre-admission visit was required. Upon entering the home the student immediately assumed the role of the nursing home resident complete with the diagnoses of dominant-side paralysis secondary to stroke and aspiration pneumonia; making them wheelchair reliant. The student remained in a wheelchair for at least 5 days and required a 1 or 2 person assist by certified nursing assistants (CNAs) when transferring from the chair to bed or toilet and to be bathed. They ate pureed foods and drank thickened liquids (for the first 24 - 36 hrs) before regaining a normal diet. Normal nursing home procedures took place that included the admissions process (forms, resident's rights, social inventory, payment review, etc.), an assessment by the nurse (vital signs, skin check, etc.) and additional assessments by an occupational therapist, physical therapist, speech and language therapist, dietary director, and activities therapist. Students were required to live, eat, sleep and "recreate" in the nursing home in which s/he was admitted for 11 days. Living in the nursing home with functional impairments ensured having multiple opportunities to experience day to day living as a resident which was captured in their journals (field notes). The Project PI maintained contact with each student during his/her stay via phone, email, texting, and visits - as a daughter might for an elder parent. The fieldwork phase ended with a debriefing session by the student and the Project PI with the nursing home administrator and staff who had regular encounters with the student. This session served the purpose of sharing experiences on what it was like to live the life of an older adult resident and was followed by an "official" discharge [1].

\subsubsection{Post-Fieldwork}

(After nursing home discharge): Upon discharge, students were driven back to the medical school and their re-entry began. Journaling continued for another 3 - 5 days (post-field notes) and addressed what it was like to leave the home and re-enter their own life; additionally they reviewed their assumptions (pre-fieldwork) and determined the accuracy of each statement based on what they learned. The Project PI conducted a final review of each journal ensuring that students answered the research question comprehensively [1].

\subsection{Data and Analyses}

Data were in the form of subjective and objective note taking referred to as journaling (pre-field notes through post-field notes). Journal writing involved presenting descriptive and detailed accounts of students' experiences, thoughts, and actions. Each entry required day, date, and time accountability, as each student wrote at varying times of the day or when presented with a significant event. Journal entries included subjective accounts_- "my feelings" as a younger person living in a nursing home-and objective accounts—-my thoughts" as a medical student. Throughout the journaling process the Project PI reviewed notes daily and feedback was provided to ensure data collection was effective and met qualitative research standards. This process also monitored each student's well being as s/he experienced this new culture. Based on past developments in qualitative ethnographic research and in this project in particular, the 10 - 12 day period assists with: 1) allowing the nursing home staff and residents to become desensitized to the new resident (the student) so that daily activities fall into routine again (days 1 - 3); 2) allowing the student to experience the changes in shifts from weekdays to weekends (days 1 - 8); 3) providing the student with feelings that they are part of the culture (days 6 - 12); and 4) permitting time to build relationships with residents in the nursing home (days 7 - 12). The journal notes taken throughout the pre-fieldwork, fieldwork, and post-fieldwork stages of the research are the key data source for Learning by Living Project. Journals were shared as a confidential document with the nursing home administrator at the close of the project [1].

Data analyses for these medical students' immersion research were conducted using basic qualitative methods for content analysis and included: 1 ) read through the journals to create an internal mind map as a starting point; 2) read through journals again to create margin notes and form initial codes/themes; 3 ) conduct open coding categories and properties; 4) performed selective coding, identifying representative quotes and listing statements of meaning for each student in relation to the research question; 5) compared coding within thematic contexts 
and intervening conditions; and 6) wrote data results based on final determination of key findings, which are presented in the results below.

\section{Results}

Four themes that intersected for the two students were: 1) control; 2) quality of life; 3) spectrum of communication; and 4) feelings of isolation. Each theme presented contains student representative quotes and reflections on these experiences.

\subsection{Theme 1: Control}

"Perhaps because of the vulnerable (read: naked) position I was in, I felt that loss of control more acutely during showering... I felt very withdrawn during this experience; like I really wanted to just pull inside myself. My voice got quieter. I spoke less. I shook it off soon, as soon as I got into the dining area for breakfast, but it was one of the more challenging experiences so far" (Philip, OMS II).

Philip's “diagnosis” required him to seek assistance with even the most basic of activities (e.g. toileting, bathing, and transferring). He witnessed a shrinking feeling that he was not familiar with and wondered how the other residents felt when they first experienced this. Recovering from this initial discomfort required a shift of perspective; a reminder to himself that this was temporary and it was important for him to experience this to gain an understanding of life lived as an older adult nursing home resident. He couldn't help but wonder what it would be like to experience this on a regular basis. Would he eventually regain his sense of control, or at the very least redefine control? A key factor that contributed to a sense of comfort was the demeanor of the staff who worked with him at these times. Their level of professionalism, compassion and empathy made these vulnerable moments more bearable.

As Philip met other residents and learned a bit about their histories he encountered Jim (pseudonym) who now lived in the nursing home due to a stroke. Philip mused in his notes: "Jim owned a business before his stroke; and stated to me that he was used to being the one calling the shots and now everyone's waiting on him and he has to rely on them.' Jim is experiencing a lot of the same frustration and awkwardness that I am..." (Philip, OMS II)

The knowledge that each resident was subjected to new limits on control was a revelation. Philip was able to fully appreciate not only his own loss of agency, but the fact that his fellow residents were experiencing this in an even more real way. Unlike him, Philip’s friend Jim didn't “... have a time limit on how long he'll be here, no time when he can just 'turn off' his condition" (Philip, OMS II).

In time, Philip became somewhat more accustomed to this limited degree of control. In learning to take control where he could, and be comfortable relinquishing it when he could not, Philip became more able to empathize with the residents' processes of finding that same balance.

"I think fear triggers the need for control, and the need for control then makes it easier to be scared. Understanding that control isn't something to strive for in an independent sort of way is, I think, the key to mitigating fear. Because in any given situation, there are a lot of pieces at play, and expecting to have control over all of them isn't realistic. For instance...some items I rely on came from somewhere beyond my personal control and looking at things in this way helps reduce the fear of losing control, by working harder to understand the full scope of the situation from a different perspective" (Ianna, OMS II).

While living in the nursing home, coming to terms with her relationship with control was a major struggle for Ianna. At one point, with the support of the Project PI, Ianna's introspection brought her a realization that control is an internal frame of mind and a belief in self-if you have inner peace, no one can disturb you unless you allow them. Eventually, Ianna was able to reframe her issues in a way that she could feel peace inside. She figured out how to manage and even be happy about living in a nursing home by focusing on personal growth. Having that experience helped her see a huge positive aspect to being a patient, while still recognizing that she had needed a catalyst - her professor - to be able to make that shift. Expressing and feeling empathy for someone else's struggle relies on knowing oneself and recognizing that personal growth is an individual thing. As a future physician, Ianna hopes to remember her struggles as an nursing home resident and be a catalyst for personal growth in her patients. 


\subsection{Theme 2: Quality of Life}

Philip lived in a locked dementia unit for 11 days. Along with being wheelchair reliant he also had to eat pureed foods (the thickness of honey) to address swallowing issues he had from his second diagnosis of pneumonia secondary to aspiration. He was upgraded to solid foods after 3 days, warranting this major breakthrough comment: “Breakfast was a victory because I got to eat solid food!” (Philip, OMS II).

Philip's experience exposed him to residents who were receiving varying levels of care, many requiring full assistance. In the beginning of his nursing home stay Philip's physical function was quite compromised, and he quickly realized how he took for granted all his prior abilities. He then gained an appreciation for those very little things he could do independently, like chewing and swallowing solid food. At first he associated his areas of inability (and those of the residents) with decreased quality of life and learned through this project that actually it was those small gains, even though not abundant, contributed to feelings of great triumph and quality of life.

Ianna's immersion in the nursing home went smoothly at first, but within a few days she was quite aware of the inherent challenges of living with significant physical function deficits and the need to rely on others for assistance. "I felt like my day had dwindled down to a couple frustrating, tired, confusing hours, which felt like a ridiculous quality of life, and not necessarily one worth sustaining” (Ianna, OMS II). Ianna began to read the environment in an effort to understand her own feelings. "He seems to look pretty sad sometimes, but it's not for me to say that he doesn't have a quality of life that he's happy with, or that I'm just catching him on a bad day. Life here is very different, and with that needs to come room for different measurements of quality of life, and happiness with life. I am a 25 year old, with much less life experience than these people have, and so my mindset is not the same; no one's mindset is exactly the same as anyone else's and that truly is worthy of a daily reminder" (Ianna, OMS II). Ianna realized the importance of having conversations with her fellow residence to understand what they were experiencing, how they viewed these experiences, and what each resident needed to meet their own definition of quality of life.

As future physicians, these students learned that communication and listening will be essential in establishing an understanding of their patients' definition of quality of life. As a result of Ianna's and Philip's experiences and reflections, they now have a much broader definition of quality of life from when they entered each nursing home. Small successes made a huge difference and realizing that each person gets to define quality of life moment by moment was a huge step towards learning about person-centered care.

\subsection{Theme 3: The Spectrum of Communication}

Philip shared a triple room, with beds on either side of his, forcing him to engage with his roommates as soon as he awoke. As he wheeled down the hallway, he encountered many residents. He became very aware of the many forms that communication can take and was excited with the progress he made in communicating with his floor mates. "Every now and again, she puts out a fully lucid sentence, or at least the beginning of an idea, and you can tell she has things to say if she can just get them out there...It becomes more about reading her face, because sometimes she'll say "Right" or "Yes," but be furrowing her brow or shaking her head" (Philip, OMS II).

Additionally, Philip realized that in the dementia unit routines are a form of communication. "I'm definitely getting closer to understanding the rhythm of the days here. Breakfast around 8. Lunch around 11, 11:30. Visitors at one, activity at two (I missed bingo today due to a PT session), dinner at quarter to five. What's different, I guess, is the rigidity of these times and routines. The residents use these as their guidance points through their day" (Philip, OMS II). He realized that these routines created meaning; a form of communication to let the residents know all was as it should be. Being the "new kid on the block” gave Philip an outsider's perspective to recognize these patterns and what the routines conveyed to the residents. Empathy, in this case, included understanding the residents' environment providing him with insights to aid these routines which helped to ground the residents in their daily lives.

Ianna was cognizant of the varied forms of verbal and non-verbal communication that were taking place in the nursing home; but her acuity regarding non-verbal communication was heightened. "She held my hand, and hers were so soft. She had deep wrinkles and happy eyes and a bright smile, a couple discolorations on her teeth here and there-some metalwork, some missing. But she had a face that was beautiful for the joy that it exuded, and because she was so kind-hearted, it didn't matter what her teeth looked like, her smile would always be gorgeous... She seemed so free and so honest with herself, with absolutely no boundaries being held up... Seeing her brightened my day. Seeing her felt like meeting someone who is a complete embodiment of being the change 
you wish to see in the world; she was an inspiring person. So I was incredibly glad to have gotten to know someone who changed my perspective on dementia, and made it so much more of an individually-based illness, or in her case, a blessing" (Ianna, OMS II).

Of note for both students was that they were better able to communicate with various residents as time went on. They used patience, eye contact, touch, and body language to exhibit empathy and support to foster communication. This illuminated that communication encompasses a full spectrum and a many-layered approach to weave a pattern of understanding, or at the very least, a feeling of engagement and respect.

\subsection{Theme 4: Isolation}

Ianna and Philip each experienced isolation in the true sense of the word of feeling separate from others especially early on in the nursing home environment—nights felt long and lonely, friends didn't come visit, mealtime conversations were sometimes non-existent, etc. But the quotes below revealed how the students experienced isolation in divergent ways. Ianna went from feeling totally isolated regardless of who she met and interacted with to then allowing herself to feel part of the community. Philip started out being overly invested in those around him to then discover the importance of establishing boundaries in order to create time of one's own.

"The frustrations broke me down, and I couldn't stop crying for the better half of the day. But being surrounded by support and understanding really helped buoy me up, and I can see that may be a huge positive side to being in a nursing home-compassion and companionship. It reminds me of a quote from Into the Wild, "happiness is only real when shared." Of course, being happy is a mindset, but having the support and interaction with other people is what makes us human. Being sick can be a very isolating thing, and a nursing home helps make that feel less isolating." (Ianna, OMS II)

"This evening I just kind of took some time for myself, and I really think it helped me recharge my batteries, so to speak...I think a good coping mechanism for me is just to take that time away. Generally, I'm fairly extroverted, and I think I've probably been riding that wave earlier on in my stay with all the new people I've been meeting and chatting with. I basically just needed to pump the breaks on that and take some alone time to regroup." (Philip, OMS II)

Physicians need to be aware of their own needs and their own sense of balance, and from this state of knowing, be willing to probe their patients to see whether the patient is maintaining a healthy balance in this regard. Additionally, nursing homes are often saddled with the belief that isolation is prevalent and the nursing home "world" is insular. Regardless of these belief systems, physicians need to be sensitive to the vastly interconnected environment in which their patients live; realizing that some patients will either choose to not engage or not have the abilities to engage.

Both students stated they gained insights into resident life that led to deeper understandings of what it can be like to live in a nursing home. As a result, they recognized the role of empathy in contributing to a caring community.

\section{Discussion}

Living the life of an older adult nursing home resident for 11 days was "life altering." The experiences, challenges, insights, emotions, skills, knowledge, patterns, and attitudes that pulsated throughout each day went far beyond anything that could ever be taught in a classroom setting. Wheeling in the chairs of the residents and living as a resident has a way of expanding perspectives about nursing home living.

Learning by Living allowed the medical students to live the life of an older adult nursing home resident complete with diagnoses and standards procedures of care. While it is useful to future physicians to be told about the experience of living in a nursing home (and even this opportunity is not universally available), actually living this experience provided far more memorable insights into these patients' daily lives. The time spent in the nursing home allowed each student to learn about both the older adult residents with whom they lived, and his or her own reactions to daily life in this unique healthcare community. In addition, one of the challenges of nursing home living that the students faced was a sense of the nursing home community being an entity unto itself: there were times where each student felt isolated from the "outside" social world, even if they had occasional visitors. The Learning by Living program, therefore, provides a unique opportunity for the students to learn from the old- 
er adult residents. Continuous time spent in this community allowed the students to be, in a limited way, insiders, and this built trust and mutual respect that created intimacy and friendship regardless of the residents' physical and/or cognitive function. Sharing feelings of loneliness with those you live with is an example of shared intimacy and one learns that those around you truly care and understand.

\section{Study Limitations}

Perhaps the greatest limitation this study is that the student researchers are at least 40 - 60 years younger than the other nursing home residents and their diagnoses were self-imposed. The degree of immersion a participant can achieve in this program is partly dependent on a willingness to commit to a kind of role-play of their given diagnoses. This highlights the importance of the pre-fieldwork and meetings with the Project PI. These students required some coaching before being able to get the full value of this kind of immersive research, and the PI is an important part of assessing the readiness of these students. The diagnoses are designed for the staff rather than the student. The staff knows how to work with a person that has dominant side weakness, for example. The standard procedures of care for toileting, bathing, feeding, transferring apply. However, as the student has no idea what it is like to live with dominant side weakness the staff needs to be willing to treat the student similarly to their older adult patients despite apparent differences between the two. Most staff provided care to the student as they would a nursing home resident. Actually, because of this project, many of the staff realized the importance of resident dignity; a point they had forgotten because they learned to focus on the job rather than on the person they cared for. In this way, the student and staff create relationships that enhance relationship building, empathy and patient-centered care.

Despite these limitations, however, living the life of an older adult resident illuminated to the student participants what it is like to be a patient. Their personal responses to issues of control, quality of life, communication, and feelings of isolation were found to be similar to the nursing home residents they befriended. Along with the students' vast spectrum of human experiences that qualified this research, they agreed that humility was the key to empathy. The word empathy was no longer a term in the dictionary, it became the lens from which they viewed care. The realization that each person exhibits unique ways of adapting instilled in the students that have a responsibility to individualize care.

\section{Conclusion}

Learning by Living nursing home immersion research is a unique project in the United States and globally. Medical students who volunteered to give up their notion of independence to challenge themselves so completely for such an extended period of time was life altering. The goal to be a better physician pressed these students to accept and adapt to limitations, self reflect on their experiences, and manage many unknowns, which led to a heartfelt understanding of empathy. Learning by Living busted the boundaries of traditional medical education and provided tools to build a bridge, connecting understanding with practice- two traits that have been shown to promote positive clinical outcomes. This project enlightened the medical students' about aging, dignity, and the importance of making meaningful connections in order to thrive. These insights translate to a comprehensive and empathic patient-centered care model, hopefully withstanding the test of time.

\section{Acknowledgements}

A special thank you is extended to the two nursing home administrators who volunteered to "admit" the student authors into their homes and the staff in each home that provided a full range of resident care so that the students could experience life lived as an elder nursing home resident.

\section{References}

[1] Gugliucci, M.R. and Weiner, A. (2012) Learning by Living: Life Altering Medical Education through Nursing Home Based Experiential Learning, Journal of Gerontology \& Geriatrics Education, 34, 60-77.

[2] White, H.K. (2008) The Nursing Home in Long-Term Care Education. Journal of the American Directors Association, 9, 75-81. http://dx.doi.org/10.1016/j.jamda.2007.11.009

[3] Denzin, N.K. and Lincoln Yvonna, S. (1994) Handbook of Qualitative Research. Sage Publications, Thousand Oaks.

[4] Denzin, N.K. and Lincoln, Y., Eds. (2005) The SAGE handbook of qualitative research. 3rd Edition. Sage, Thousand 
Oaks.

[5] Calabrese, L., Bianco, J., Mann, D., Massello, D. and Hojat, M. (2013) Correlates and Changes in Empathy and Attitudes Toward Interprofessional Collaboration in Osteopathic Medical Students. The Journal of the American Osteopathic Association, 18, 898-907. http://dx.doi.org/10.7556/jaoa.2013.068

[6] Reynolds, W. and Scott, B. (1999) Empathy: A Crucial Component of the Helping Relationship. Journal of Psychiatric and Mental Health Nursing, 6, 363-370. http://dx.doi.org/10.1046/j.1365-2850.1999.00228.x

[7] Glaser, B.G. (1978) Theoretical Sensitivity. Sociological Press, Mill Valley.

[8] Glaser, B.G. and Strauss, A.L. (1987) The Discovery of Grounded Theory: Strategies of Qualitative Research. Aldine, New York.

[9] Strauss, A. and Corbin, J. (1994) Grounded Theory Methodology: An Overview. In: Denzin, N.K. and Lincoln Yvonna, S., Eds., Handbook of Qualitative Research, Sage Publications, Thousand Oaks, 273-285.

[10] Holstein, J.A. and Gubrium, J.F. (1994) Phenomenology, Ethnomethodology, and Interpretive Practice. In: Denzin, N.K. and Lincoln Yvonna, S., Eds., Handbook of Qualitative Research, Sage Publications. Thousand Oaks, 262-272. 